TRANSACTIONS OF THE

AMERICAN MATHEMATICAL SOCIETY

Volume 348, Number 5, May 1996

\title{
DECOMPOSITIONS IN QUANTUM LOGIC
}

\author{
JOHN HARDING
}

\begin{abstract}
We present a method of constructing an orthomodular poset from a relation algebra. This technique is used to show that the decompositions of any algebraic, topological, or relational structure naturally form an orthomodular poset, thereby explaining the source of orthomodularity in the ortholattice of closed subspaces of a Hilbert space. Several known methods of producing orthomodular posets are shown to be special cases of this result. These include the construction of an orthomodular poset from a modular lattice and the construction of an orthomodular poset from the idempotents of a ring.

Particular attention is paid to decompositions of groups and modules. We develop the notion of a norm on a group with operators and of a projection on such a normed group. We show that the projections of a normed group with operators form an orthomodular poset with a full set of states. If the group is abelian and complete under the metric induced by the norm, the projections form a $\sigma$-complete orthomodular poset with a full set of countably additive states.

We also describe some properties special to those orthomodular posets constructed from relation algebras. These properties are used to give an example of an orthomodular poset which cannot be embedded into such a relational orthomodular poset, or into an orthomodular lattice. It had previously been an open question whether every orthomodular poset could be embedded into an orthomodular lattice.
\end{abstract}

\section{INTRODUCTION}

A cornerstone of the quantum logic approach to quantum mechanics is the fact that the closed subspaces of a Hilbert space satisfy the orthomodular law

$$
\text { if } A \subseteq B \text {, then } B=A \oplus\left(A^{\perp} \cap B\right) \text {. }
$$

There has long been a question as to why a Hilbert space should be the vehicle used to describe quantum mechanics, so it is natural to ask whether the axioms of a Hilbert space could somehow be weakened while retaining orthomodularity. A theorem of Amemiya and Araki [1] shows that the ortholattice of closed subspaces of a Euclidean space is orthomodular if and only if the Euclidean space is complete, i.e. a Hilbert space. So at first glance it seems that orthomodularity is intricately tied to the structure of the Hilbert space and will not be preserved by any useful weakening of the axioms. This could not be further from the truth.

Received by the editors November 22, 1994.

1991 Mathematics Subject Classification. Primary 81P10, 06Cxx, 03G15.

Key words and phrases. Hilbert space, normed group, orthomodular poset, projection operator, quantum logic, relation algebra.

Research supported by the Natural Sciences and Engineering Research Council of Canada.

(C)1996 American Mathematical Society 
We show that absolutely none of the structure of a Hilbert space is necessary to produce an orthomodular poset. One must simply adjust the way in which closed subspaces are viewed. The point is that closed subspaces are in complete correspondence with decompositions $\mathcal{H} \cong \mathcal{H}_{1} \times \mathcal{H}_{2}$ of the Hilbert space $\mathcal{H}$ into the product of two Hilbert spaces $\mathcal{H}_{1}$ and $\mathcal{H}_{2}$. With each such decomposition we associate an ordered pair of equivalence relations $\left(\alpha_{1}, \alpha_{2}\right)$, where $\alpha_{i}$ is the kernel of the projection onto $\mathcal{H}_{i}$. We can then view the orthomodular poset of closed subspaces of a Hilbert space as being constructed from certain ordered pairs of equivalence relations on $\mathcal{H}$.

We can mimic this procedure for any set $X$. We define Fact $X$ to be those pairs of equivalence relations $\left(\alpha_{1}, \alpha_{2}\right)$ on $X$ which occur as the kernels of the projections associated with a direct decomposition $X \cong X_{1} \times X_{2}$; two such pairs of equivalence relations are orthogonal if their associated decompositions admit a common refinement. As we shall see, Fact $X$ carries the structure of an orthomodular poset. Therefore, orthomodularity is not due to any intricate structure of the Hilbert space - it is a consequence of arithmetical properties of relations.

This paper is organized in the following manner. Section 2 contains a brief introduction to relation algebras. Relation algebras are an abstraction of the algebra $R X$ of all relations on a set $X$, and it should not be surprising that our construction of an orthomodular poset from the relations on a set $X$ can be extended to an arbitrary relation algebra. All of our results remain valid in this more general setting, and will be presented in this way. This extra generality is worthwhile, as it allows a unified presentation of several techniques for constructing orthomodular posets. The reader who has no interest in relation algebras can read the great majority of this paper simply by considering our results in the familiar setting of relations over a set. However, it is still advisable to read Section 2 in order to familiarize oneself with our notation for relational products, etc.

The central result of the paper is contained in Section 3; here we describe how one can construct an orthomodular poset from any relation algebra. In Section 4 we give examples of orthomodular posets constructed from relation algebras. These examples include many familiar orthomodular posets such as the projection operators of a Hilbert space, all modular ortholattices, orthomodular posets constructed from modular lattices [18], and orthomodular posets constructed from the idempotents of a ring with unit $[7,14]$. New examples of orthomodular posets are also given. We show that the decompositions of any algebraic, topological or relational structure form an orthomodular poset, and show how to construct an orthomodular poset from the subgroups of a group.

In Section 5 we focus our results on decompositions of algebraic structures to groups with operators, a class of algebras which includes groups and modules. There are alternate ways of describing the decompositions of a group $G$ with operators which are better suited to calculations; we can describe the decompositions of $G$ in terms of inner direct sums, or by using certain idempotent endomorphisms of $G$. The structure of the orthomodular poset Fact $G$ is described both in terms of inner direct sums and in terms of endomorphisms of $G$, laying the groundwork for results in Section 6. The description of Fact $G$ in terms of endomorphisms of $G$ is closely related to the logic of idempotents of a ring as studied by Flachsmeyer [7] and Katrnoška [14].

In Section 6 we continue our investigation into the decompositions of a group with operators. We define the notion of a norm on a group with operators, and of a 
projection on a normed group with operators. We show that these projections have many of the properties of projection operators on a Hilbert space. In particular, the projection operators of a normed group form an orthomodular poset with a full set of states, and the projection operators of a complete normed abelian group form a $\sigma$-complete orthomodular poset with a full set of countably additive states.

In the final section, we give examples of orthomodular posets which cannot be embedded into any relational orthomodular poset. We also show that these pathological orthomodular posets cannot be embedded into any orthomodular lattice. The question as to whether every orthomodular poset could be embedded into an orthomodular lattice surprisingly seems to have been open.

\section{Relation ALGEBRAS}

Relation algebras were first introduced by Tarski in [20]. The motivating example is the algebra $R X$ of all relations over a set $X$. Specifically, this consists of the Boolean algebra $B$ of all subsets of $X \times X$ together with the operation ; of relational multiplication, $\smile$ of conversion, and the constant $1^{\prime}$ being the identical relation. The reader should consult [11] for background on relation algebras.

Definition 2.1. A relation algebra is a Boolean algebra $(B,+, \cdot,-, 0,1)$ with an additional binary operation ; , an additional unary operation $\smile$ and a constant $1^{\prime}$, which satisfies the following identities:

(2.1.1) $(a ; b) ; c=a ;(b ; c)$,

(2.1.2) $(a+b) ; c=a ; c+b ; c$,

(2.1.3) $a ; 1^{\prime}=a$,

(2.1.4) $a^{\smile}=a$,

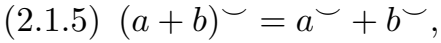

(2.1.6) $(a ; b)^{\smile}=b^{\smile} ; a^{\smile}$

$(2.1 .7) a^{-} ;(a ; b)^{-}+b^{-}=b^{-}$.

This system of axioms was chosen to be satisfied in any algebra $R X$. However, there are identities which hold in each algebra $R X$ which are not implied by this axiom system [11]. Therefore, relation algebras are a true generalization of the algebras of relations over a set.

The following lemma, due to Chin and Tarski [5], states that a fragment of modularity holds in any relation algebra. This is a key point in our study of decompositions.

Lemma 2.2. Let $a, b, c$ be elements of a relation algebra $R$.

(2.2.1) If $a ; c \leq a$ and $a ; c^{\smile} \leq a$, then $a \cdot(b ; c)=(a \cdot b) ; c$.

(2.2.2) If $c ; a \leq a$ and $c^{-} ; a \leq a$, then $(c ; b) \cdot a=c ;(b \cdot a)$.

(2.2.3) If $c=c^{\smile}$ and $a ; c=c ; a=a$ and $a \leq b ; c=c ; b$, then $(a \cdot b) ; c=a=$ $c ;(b \cdot a)$.

(2.2.4) If $c \leq a=a^{\smile}=a ; a$ and $a \cdot b=1^{\prime}$, then $a \cdot(c ; b)=c$.

Proof. The first statement can be found in [5, Corollary 2.19]. The second statement follows from the first using (2.1.4), (2.1.6) and the fact that $(a \cdot b)^{\smile}=a^{\smile} \cdot b^{\smile}$. The third statement follows directly from the first two, and the fourth follows from the second using (2.1.3). 


\section{ORTHOMODULAR POSETS FROM RELATION ALGEBRAS}

We will describe a method to construct an orthomodular poset from a relation algebra. The reader will recall that an orthocomplemented poset is a structure $(P, \leq, 0,1, \perp)$, where $(P, \leq, 0,1)$ is a bounded poset and $\perp$ is an order inverting map of period two such that $x^{\perp}$ is a complement of $x$ for each $x \in P$. An orthocomplemented poset is an orthomodular poset if for each pair of elements $x, y \in P$ with $x \leq y^{\perp}$ we have that the join $x+y$ of $x$ and $y$ exists and $y^{\perp}=x+(x+y)^{\perp}$. This latter condition is referred to as the orthomodular law. The reader should see $[2,13,19]$ for a complete account of orthomodular posets and their applications to quantum mechanics.

Definition 3.1. For a relation algebra $R$ define

(3.1.1) $R^{(1)}=\left\{x \in R: 1^{\prime} \leq x=x ; x=x^{\smile}\right\}$,

(3.1.2) $R^{(2)}=\left\{\left(x, x^{\prime}\right) \in R^{(1)} \times R^{(1)}: 1^{\prime}=x \cdot x^{\prime}\right.$ and $\left.x ; x^{\prime}=x^{\prime} ; x=1\right\}$.

And define a relation $\subseteq$ on $R^{(2)}$ by

(3.1.3) $\left(x, x^{\prime}\right) \subseteq\left(y, y^{\prime}\right)$ if $x \leq y, y^{\prime} \leq x^{\prime}$ and $x ; y^{\prime}=y^{\prime} ; x$.

We shall call the members of $R^{(1)}$ proper equivalence elements. The term equivalence element has historically been used without the requirement that $1^{\prime} \leq x$.

Remark 3.2. For the relation algebra $R X$ of all binary relations on a set $X, R X^{(1)}$ is just the set of all equivalence relations on $X$, and $R X^{(2)}$ is the set of all ordered pairs of equivalence relations $\left(x, x^{\prime}\right)$ such that $X \cong X / x \times X / x^{\prime}$. For the relation algebra $R X$, the content of the following lemma is that $\left(x, x^{\prime}\right) \subseteq\left(y, y^{\prime}\right)$ if and only if $X$ is canonically isomorphic to $X / y \times X /\left(x ; y^{\prime}\right) \times X / x^{\prime}$. In other words, $\left(x, x^{\prime}\right) \subseteq\left(y, y^{\prime}\right)$ if the decompositions $X \cong X / x \times X / x^{\prime}$ and $X \cong X / y^{\prime} \times X / y$ admit a common refinement.

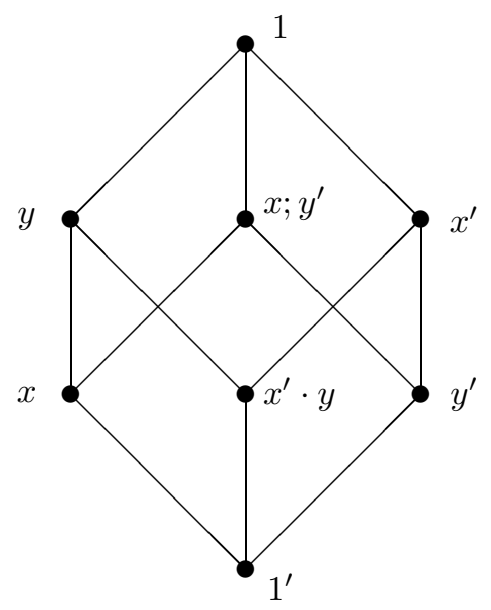

Lemma 3.3. Let $\left(x, x^{\prime}\right)$ and $\left(y, y^{\prime}\right)$ be elements of $R^{(2)}$ for some relation algebra R. If $\left(x, x^{\prime}\right) \subseteq\left(y, y^{\prime}\right)$, then

(3.3.1) $x ;\left(x^{\prime} \cdot y\right)=y=\left(x^{\prime} \cdot y\right) ; x$,

(3.3.2) $y^{\prime} ;\left(x^{\prime} \cdot y\right)=x^{\prime}=\left(x^{\prime} \cdot y\right) ; y^{\prime}$, 
(3.3.3) $y \cdot\left(x ; y^{\prime}\right)=x$,

(3.3.4) $x^{\prime} \cdot\left(x ; y^{\prime}\right)=y^{\prime}$.

This implies that the set $\left\{1^{\prime}, 1, x, x^{\prime}, y, y^{\prime},\left(x^{\prime} \cdot y\right),\left(x ; y^{\prime}\right)\right\}$ is a Boolean lattice under the partial ordering inherited from $R$. Meets in this lattice agree with meets in $R$, while joins are given by $;.$ In particular, the elements of this set commute under the operation;.

Proof. Note first that for elements $a, b$ of $R^{(1)}$, if $a \leq b$, then $a ; b=b ; a=b$. We make repeated use of this fact in conjunction with the assumptions $x \leq y$ and $y^{\prime} \leq x^{\prime}$. The first statement follows directly from (2.2.3) using $y, x^{\prime}$ and $x$ in place of $a, b$ and $c$ respectively. The second statement also follows from (2.2.3) using $x^{\prime}$, $y$ and $y^{\prime}$ in place of $a, b$ and $c$ respectively. The third statement follows from (2.2.2) using $y, y^{\prime}$ and $x$ in place of $a, b$ and $c$ respectively (recall that $y \cdot y^{\prime}=1^{\prime}$ since $\left(y, y^{\prime}\right)$ is in $\left.R^{(2)}\right)$. The fourth statement follows from (2.2.2) using $x^{\prime}, x$ and $y^{\prime}$ in place of $a, b$ and $c$ respectively.

These four conditions and the assumption that $\left(x, x^{\prime}\right) \subseteq\left(y, y^{\prime}\right)$ imply that the set $\left\{1^{\prime}, 1, x, x^{\prime}, y, y^{\prime},\left(x^{\prime} \cdot y\right),\left(x ; y^{\prime}\right)\right\}$ is closed under the operations · and ;. Therefore this set forms a lattice under the partial ordering inherited from $R$, with meets agreeing with those in $R$. That joins are given by ; follows from the monotonicity of ;. It is then routine to check that this lattice is Boolean.

Definition 3.4. For a relation algebra $R$, define a map $\perp: R^{(2)} \longrightarrow R^{(2)}$ and constants $\underline{0}$ and $\underline{1}$ of $R^{(2)}$ as follows:

(3.4.1) $\left(x, x^{\prime}\right)^{\perp}=\left(x^{\prime}, x\right)$,

(3.4.2) $\underline{0}=\left(1^{\prime}, 1\right)$,

(3.4.3) $\underline{1}=\left(1,1^{\prime}\right)$.

Theorem 3.5. For any relation algebra $R$, the system $\left(R^{(2)}, \subseteq, \underline{0}, \underline{1}, \perp\right)$ is an orthomodular poset. The operation $\oplus$ of orthogonal join in $R^{(2)}$ is given as follows:

(3.5.1) if $\left(x, x^{\prime}\right) \subseteq\left(y, y^{\prime}\right)^{\perp}$, then $\left(x, x^{\prime}\right) \oplus\left(y, y^{\prime}\right)=\left((x ; y),\left(x^{\prime} \cdot y^{\prime}\right)\right)$.

Proof. We first show that $\subseteq$ is a partial ordering. The only point which is not obvious is transitivity. If $\left(x, x^{\prime}\right) \subseteq\left(y, y^{\prime}\right) \subseteq\left(z, z^{\prime}\right)$, we must show that $\left(x, x^{\prime}\right) \subseteq$ $\left(z, z^{\prime}\right)$. This will follow easily once we have shown that $x ; z^{\prime}=z^{\prime} ; x$. Note that by Lemma 3.3

$$
z^{\prime} \leq\left(x ; z^{\prime} ; x\right) \cdot y^{\prime} \leq\left(y ; z^{\prime} ; y\right) \cdot y^{\prime}=\left(y ; z^{\prime}\right) \cdot y^{\prime}=z^{\prime} .
$$

So $\left(x ; z^{\prime} ; x\right) \cdot y^{\prime}=z^{\prime}$. By applying $(2.2 .3)$ with $\left(x ; z^{\prime} ; x\right), y^{\prime}$ and $x$ in place of $a, b$ and $c$ respectively, we obtain

$$
x ;\left(\left(x ; z^{\prime} ; x\right) \cdot y^{\prime}\right)=\left(\left(x ; z^{\prime} ; x\right) \cdot y^{\prime}\right) ; x .
$$

Upon substituting $\left(x ; z^{\prime} ; x\right) \cdot y^{\prime}=z^{\prime}$, we have $x ; z^{\prime}=z^{\prime} ; x$ as required.

It is easily seen that $\underline{0}$ and $\underline{1}$ are the lower and upper bounds, respectively, of the poset $R^{(2)}$. It is also clear that $\perp$ is an orthocomplementation. We next verify (3.5.1). If $\left(x, x^{\prime}\right) \subseteq\left(y, y^{\prime}\right)^{\perp}$, then by Lemma 3.3, $\left((x ; y),\left(x^{\prime} \cdot y^{\prime}\right)\right)$ is in $R^{(2)}$ and is clearly the least upper bound of $\left(x, x^{\prime}\right)$ and $\left(y, y^{\prime}\right)$. We have only to check the orthomodular law. If $\left(x, x^{\prime}\right) \subseteq\left(y, y^{\prime}\right)$, then by (3.5.1)

$$
\left(x, x^{\prime}\right) \oplus\left(\left(x, x^{\prime}\right) \oplus\left(y, y^{\prime}\right)^{\perp}\right)^{\perp}=\left(\left(x ;\left(x^{\prime} \cdot y\right)\right),\left(x^{\prime} \cdot\left(x ; y^{\prime}\right)\right)\right) .
$$

Then an application of Lemma 3.3 concludes the proof. 


\section{Relational orthomodular posets}

An orthomodular poset $P$ will be called a relational orthomodular poset if there is a relation algebra $R$ and an order embedding of $P$ into $R^{(2)}$ which preserves orthocomplementation and finite orthogonal joins. We show that the orthomodular lattice $\mathcal{L}(\mathcal{H})$ of closed subspaces of a Hilbert space $\mathcal{H}$ is a relational orthomodular poset. In fact, it is a sub-orthomodular poset of $R H^{(2)}$, the collection of all decompositions of the underlying set of $\mathcal{H}$. This demonstrates that the orthomodularity in $\mathcal{L}(\mathcal{H})$ is due to arithmetical properties of relations. In this section, we will describe several methods for constructing relational orthomodular posets. In particular, we show that the decompositions of any algebraic, relational or topological structure form a relational orthomodular poset.

Remark 4.1. For a set $X$, the collection of binary relations over $X$ forms a relation algebra $R X$. It is easily seen that $R X^{(2)}$ consists of exactly those pairs of equivalence relations $(\alpha, \beta)$ for which the natural map from $X$ to $X / \alpha \times X / \beta$ is an isomorphism. Note that the condition for $(\alpha, \beta)$ to be orthogonal to $\left(\alpha^{\prime}, \beta^{\prime}\right)$ amounts to saying that

$$
X \cong X / \beta \times X /\left(\alpha ; \alpha^{\prime}\right) \times X / \beta^{\prime}
$$

is a common refinement of the decompositions $X \cong X / \alpha \times X / \beta$ and $X \cong X / \alpha^{\prime} \times$ $X / \beta^{\prime}$. To be consistent with the notation of Section 3 , we use; to denote relational product and $\cdot$ for the intersection of relations.

Remark 4.2. For an algebra $A=\left(A,\left(f_{i}\right)_{I}\right)$, let Fact $A$ be the set of all pairs $(\alpha, \beta)$ in $R A^{(2)}$ for which $\alpha$ and $\beta$ are congruences on $A$. Clearly Fact $A$ is closed under the orthocomplementation of $R A^{(2)}$. But the intersection of two congruences is a congruence, and the relational product of permuting congruences is a congruence. It follows that Fact $A$ is closed under finite orthogonal joins taken in $R A^{(2)}$. Therefore, Fact $A$ is a relational orthomodular poset. Notice that these remarks remain valid even if we allow $A$ to have infinitary operations.

Definition 4.3. Let $(X, \tau)$ be a topological space, where $\tau$ denotes the collection of open subsets of $X$. If $\alpha$ is an equivalence relation on $X$, we define $\tau / \alpha$ to be a collection of subsets of $X / \alpha$ by setting

$$
\text { (4.3.1) } \tau / \alpha=\{\{x / \alpha: x \in A\}: A \in \tau\} \text {. }
$$

If $(\alpha, \beta)$ is a pair of equivalence relations in $R X^{(2)}$ we write $\tau=\tau / \alpha \times \tau / \beta$ if $\tau / \alpha$ and $\tau / \beta$ are topologies and the space $(X, \tau)$ is naturally homeomorphic to the product of the spaces $(X / \alpha, \tau / \alpha)$ and $(X / \beta, \tau / \beta)$. There may well be no topologies on $X / \alpha$ and $X / \beta$ making $(X, \tau)$ naturally homeomorphic to the product of the spaces $X / \alpha$ and $X / \beta$. However, if such topologies do exist, the fact that projection maps are open and continuous implies that these topologies must be equal to $\tau / \alpha$ and $\tau / \beta$. Define

(4.3.2) Fact $(X, \tau)=\left\{(\alpha, \beta) \in R X^{(2)}: \tau=\tau / \alpha \times \tau / \beta\right\}$.

Theorem 4.4. If $(X, \tau)$ is a topological space, then Fact $(X, \tau)$ is a sub-orthomodular poset of $R X^{(2)}$, and therefore Fact $(X, \tau)$ is a relational orthomodular poset.

Proof. It is obvious that Fact $(X, \tau)$ is closed under orthocomplementation. We have only to show that it is closed under finite orthogonal joins. 
Let $(\alpha, \beta)$ and $\left(\alpha^{\prime}, \beta^{\prime}\right)$ be orthogonal elements of Fact $(X, \tau)$. We may assume that $X=A \times B \times C$ and that

$$
X / \beta \cong A, X /\left(\alpha ; \alpha^{\prime}\right) \cong B \text { and } X / \beta^{\prime} \cong C,
$$

while

$$
X / \alpha \cong B \times C, \quad X /\left(\beta \cdot \beta^{\prime}\right) \cong A \times C \text { and } X / \alpha^{\prime} \cong A \times B .
$$

Then as $(\alpha, \beta)$ and $\left(\alpha^{\prime}, \beta^{\prime}\right)$ are in Fact $(X, \tau)$, there are topologies $\mu, \lambda, \delta$ and $\pi$ such that

and

$$
(A \times B \times C, \tau) \cong(A, \mu) \times(B \times C, \lambda)
$$

$$
(A \times B \times C, \tau) \cong(A \times B, \delta) \times(C, \pi) .
$$

If we can show there is a topology $\xi$ on $B$ such that

$$
(A \times B \times C, \tau) \cong(A, \mu) \times(B, \xi) \times(C, \pi),
$$

it will follow that

$$
(A \times B \times C, \tau) \cong(B, \xi) \times(A \times C, \mu \times \pi),
$$

where $\mu \times \pi$ is the product topology. But this is precisely what is needed to show that $\left(\alpha ; \alpha^{\prime}, \beta \cdot \beta^{\prime}\right)$ is in Fact $(X, \tau)$.

Before we define the topology $\xi$ on $B$ required to prove the theorem, it will be convenient to establish some notation for the various projection operations. A fundamental fact that we use repeatedly is that the projection operations associated with a topological direct product are both open and continuous. Let $f, g$ and $h$ be the projections of $A \times B \times C$ onto $A, B$ and $C$ respectively, and $f \times g, f \times h$ and $g \times h$ be the projections of $A \times B \times C$ onto $A \times B, A \times C$ and $B \times C$ respectively. For any subset $Y$ of $A \times B \times C$, it is a matter of routine to verify that

$$
A \times g[Y] \times C=A \times(g \times h)\left[Y^{\prime}\right] \text {, where } Y^{\prime}=(f \times g)[Y] \times C .
$$

It follows from the fact that $\tau=\mu \times \lambda$ and $\tau=\delta \times \pi$, that if $Y$ is open in $\tau$, then $A \times g[Y] \times C$ is also open in $\tau$. Set

We are now ready to define the topology $\xi$ on $B$ needed to establish the theorem.

$$
\xi=\{g[Y]: Y \in \tau\}
$$

Clearly $\xi$ is closed under arbitrary unions. To show that $\xi$ is a topology, we must show that for all open subsets $Y_{1}$ and $Y_{2}$ in $\tau$ and all points $b$ in $B$,

$$
\text { if } b \in g\left[Y_{1}\right] \cap g\left[Y_{2}\right] \text {, then there is } Y \in \tau \text { with } b \in g[Y] \subseteq g\left[Y_{1}\right] \cap g\left[Y_{2}\right] .
$$

It is a simple matter to check that we may set $Y$ to be the intersection of $A \times g\left[Y_{1}\right] \times C$ and $A \times g\left[Y_{2}\right] \times C$. We have shown that $\xi$ is a topology on $B$.

It remains only to show that $\tau$ is equal to the product topology $\mu \times \xi \times \pi$. As $\tau=\mu \times \lambda$, it follows that $\mu=\{f[Y]: Y \in \tau\}$, and as $\tau=\delta \times \pi$, it follows that $\pi=\{h[Y]: Y \in \tau\}$. Therefore, the product topology $\mu \times \xi \times \pi$ has as a sub-basis the set

$$
\{f[Y] \times B \times C: Y \in \tau\} \cup\{A \times g[Y] \times C: Y \in \tau\} \cup\{A \times B \times h[Y]: Y \in \tau\} .
$$

As $\tau=\mu \times \lambda$, it follows that the first of these sets is in $\tau$, and as $\tau=\delta \times \pi$, it follows that the last of these sets is in $\tau$. But in an earlier remark, we have established that the second of these sets is in $\tau$. Therefore this sub-basis of the product topology is contained in $\tau$, giving that $\tau$ contains the product topology. To show that $\tau$ is contained in the product topology, assume that $\left(a_{0}, b_{0}, c_{0}\right)$ is an element of an open 
set $Y$ of $\tau$. As $\tau=\mu \times \lambda$, there is a $\tau$-open subset $U$ of $Y$ with $\left(a_{0}, b_{0}, c_{0}\right)$ in $U$ and $U=P \times Q$ for some $P \subseteq A$ and some $Q \subseteq B \times C$. Note that $U$ satisfies

$$
\text { if }(a, b, c) \in U \text { and }\left(a^{\prime}, b^{\prime}, c^{\prime}\right) \in U \text {, then }\left(a, b^{\prime}, c^{\prime}\right) \in U \text {. }
$$

But $\tau=\delta \times \pi$, so there is a $\tau$-open subset $V$ of $U$ with $\left(a_{0}, b_{0}, c_{0}\right)$ in $V$ and $V=R \times S$ for some $R \subseteq A \times B$ and $S \subseteq C$. Note that $V$ satisfies

$$
\text { if }(a, b, c) \in V \text { and }\left(a^{\prime}, b^{\prime}, c^{\prime}\right) \in V \text {, then }\left(a, b, c^{\prime}\right) \in V \text {. }
$$

Set $W=f[V] \times g[V] \times h[V]$. It is easily seen that $\left(a_{0}, b_{0}, c_{0}\right)$ is in $W$, and the properties of $U$ and $V$ listed above readily establish that $W$ is contained in $Y$. But $V$ is open in $\tau$, and this implies $W$ is open in the product topology. Therefore each point of $Y$ has a neighbourhood which is open in the product topology and contained in $Y$, so $Y$ is also open in the product topology. So $\tau=\mu \times \xi \times \pi$, which establishes the theorem.

Definition 4.5. Let $(X, T)$ be a binary relational structure with $T$ a non-empty relation on $X$. If $\alpha$ is an equivalence relation on $X$, define a binary relation $T / \alpha$ on $X / \alpha$ by setting

(4.5.1) $T / \alpha=\{(x / \alpha, y / \alpha):(x, y) \in T\}$.

If $(\alpha, \beta)$ is a pair of equivalence relations in $R X^{(2)}$, write $T=T / \alpha \times T / \beta$ if $(X, T)$ is naturally isomorphic to the product of the structures $(X / \alpha, T / \alpha)$ and $(X / \beta, T / \beta)$. There may be no relations on $X / \alpha$ and $X / \beta$ making $(X, T)$ naturally isomorphic to the product of the structures $X / \alpha$ and $X / \beta$. However, if such relations do exist, they must be equal to $T / \alpha$ and $T / \beta$. Define

(4.5.2) Fact $(X, T)=\left\{(\alpha, \beta) \in R X^{(2)}: T=T / \alpha \times T / \beta\right\}$.

Theorem 4.6. If $(X, R)$ is a binary relational structure with the relation $R$ nonempty, then Fact $(X, R)$ is a sub-orthomodular poset of $R X^{(2)}$, and therefore Fact $(X, R)$ is a relational orthomodular poset.

Proof. Proceeding as in the proof of Theorem 4.4, it is sufficient to show that if $R, S, T, U$ and $V$ are relations such that

$$
(A \times B \times C, R) \cong(A, U) \times(B \times C, V)
$$

and

$$
(A \times B \times C, R) \cong(A \times B, S) \times(C, T),
$$

then there is a relation $W$ on $B$ such that

$$
(A \times B \times C, R) \cong(A, U) \times(B, W) \times(C, T) .
$$

Note that the assumptions imply that

$$
\begin{gathered}
U=\left\{\left(a, a^{\prime}\right): \text { there exist } b, b^{\prime}, c, c^{\prime} \text { with }(a, b, c) R\left(a^{\prime}, b^{\prime}, c^{\prime}\right)\right\}, \\
V=\left\{\left((b, c),\left(b^{\prime}, c^{\prime}\right)\right): \text { there exist } a, a^{\prime} \text { with }(a, b, c) R\left(a^{\prime}, b^{\prime}, c^{\prime}\right)\right\}, \\
S=\left\{\left((a, b),\left(a^{\prime}, b^{\prime}\right)\right): \text { there exist } c, c^{\prime} \text { with }(a, b, c) R\left(a^{\prime}, b^{\prime}, c^{\prime}\right)\right\},
\end{gathered}
$$

and

$$
T=\left\{\left(c, c^{\prime}\right): \text { there exist } a, a^{\prime}, b, b^{\prime} \text { with }(a, b, c) R\left(a^{\prime}, b^{\prime}, c^{\prime}\right)\right\} .
$$

Define

$$
W=\left\{\left(b, b^{\prime}\right): \text { there exist } a, a^{\prime}, c, c^{\prime} \text { with }(a, b, c) R\left(a^{\prime}, b^{\prime}, c^{\prime}\right)\right\} .
$$


We have to show that $R$ is the product of the relations $U, W$ and $T$. For convenience, we denote this product relation by $P$. Note that

$$
P=\left\{\left((a, b, c),\left(a^{\prime}, b^{\prime}, c^{\prime}\right)\right): a U a^{\prime}, b W b^{\prime} \text { and } c T c^{\prime}\right\} .
$$

If $(a, b, c)$ is $R$-related to $\left(a^{\prime}, b^{\prime}, c^{\prime}\right)$, then we have $\left(a, a^{\prime}\right) \in U,\left(b, b^{\prime}\right) \in W$ and $\left(c, c^{\prime}\right) \in T$, and therefore $(a, b, c)$ is $P$-related to $\left(a^{\prime}, b^{\prime}, c^{\prime}\right)$. So $R$ is contained in $P$. Next, assume that $\left(a_{1}, b_{2}, c_{3}\right)$ is $P$-related to $\left(a_{1}^{\prime}, b_{2}^{\prime}, c_{3}^{\prime}\right)$. Then $\left(a_{1}, a_{1}^{\prime}\right) \in U$, $\left(b_{2}, b_{2}^{\prime}\right) \in W$ and $\left(c_{3}, c_{3}^{\prime}\right) \in T$. This implies that there are elements $a_{i}, a_{i}^{\prime}, b_{i}, b_{i}^{\prime}$ and $c_{i}, c_{i}^{\prime}$ for $i=1,2,3$ such that

$$
\left(a_{1}, b_{1}, c_{1}\right) R\left(a_{1}^{\prime}, b_{1}^{\prime}, c_{1}^{\prime}\right),\left(a_{2}, b_{2}, c_{2}\right) R\left(a_{2}^{\prime}, b_{2}^{\prime}, c_{2}^{\prime}\right) \text { and }\left(a_{3}, b_{3}, c_{3}\right) R\left(a_{3}^{\prime}, b_{3}^{\prime}, c_{3}^{\prime}\right) .
$$

Then $\left(b_{2}, c_{2}\right)$ is $V$-related to $\left(b_{2}^{\prime}, c_{2}^{\prime}\right)$ and $a_{1}$ is $U$-related to $a_{1}^{\prime}$. As $R$ is equal to the product of the relations $U$ and $V$, we have that

$$
\left(a_{1}, b_{2}, c_{2}\right) R\left(a_{1}^{\prime}, b_{2}^{\prime}, c_{2}^{\prime}\right) \text {. }
$$

This implies that $\left(a_{1}, b_{2}\right)$ is $S$-related to $\left(a_{1}^{\prime}, b_{2}^{\prime}\right)$. But $c_{3}$ is $T$-related to $c_{3}^{\prime}$, and as $R$ is equal to the product of the relations $S$ and $T$, it follows that

$$
\left(a_{1}, b_{2}, c_{3}\right) R\left(a_{1}^{\prime}, b_{2}^{\prime}, c_{3}^{\prime}\right) \text {. }
$$

Therefore $P$ is contained in $R$, giving that $P$ and $R$ are equal.

Remark 4.7. We have shown that the factor pairs of any algebra, topological space or non-empty relational structure form a sub-orthomodular poset of $R X^{(2)}$, where $X$ is the underlying set of the structure. As the intersection of a family of suborthomodular posets is a sub-orthomodular poset, we may combine these results at will. For example, the factor pairs of a partially ordered topological group would form a relational orthomodular poset.

Remark 4.8. Let $\mathcal{H}$ be a Hilbert space. For a closed subspace $A$ of $\mathcal{H}$, it is wellknown that every vector $v$ in $\mathcal{H}$ can be expressed in a unique manner as the sum of a vector in $A$ and a vector in $A^{\perp}$. If we let $A$ denote both a subspace of the vector space $\mathcal{H}$ and the congruence associated with that subspace, we have that $\left(A, A^{\perp}\right)$ is an element of $R H^{(2)}$ for every closed subspace $A$ of $\mathcal{H}$. If $A$ and $B$ are closed subspaces with $A \subseteq B^{\perp}$, then the subspace $A \oplus B$ generated by $A \cup B$ is closed and $(A \oplus B)^{\perp}$ is equal to $A^{\perp} \cap B^{\perp}$. It follows that the collection of all such pairs $\left(A, A^{\perp}\right)$ is a subset of $R H^{(2)}$ which is closed under orthocomplementation and finite orthogonal joins. Therefore the orthomodular lattice of closed subspaces of a Hilbert space is a relational orthomodular poset.

Remark 4.9. Flachsmeyer [7] and Katrnoška [14] introduced a method to construct an orthomodular poset $\mathcal{L} A$ from the idempotents of a $\operatorname{ring} A$ with unit. For idempotents $e$ and $f$, set $e \leq f$ if $e f=e=f e$, and put $e^{\perp}=1-e$. As idempotents of $A$ correspond to direct decompositions of the left $A$-module $A_{A}$, it follows that $\mathcal{L} A$ is isomorphic to the relational orthomodular poset Fact $A_{A}$. We leave it to the reader to verify that $e \sim(\alpha, \beta)$ is the required isomorphism, where $\alpha=\left\{(x, y) \in A^{2}: x-y \in A e\right\}$ and $\beta=\left\{(x, y) \in A^{2}: x-y \in A(1-e)\right\}$. See also Remark 5.8.

Remark 4.10. An interesting method of constructing relational orthomodular posets comes from the fact that the complex algebra of a group $G$ is a relation algebra $G^{+}[11]$. The elements of $G^{+}$are subsets of $G$, the relational product of two subsets $A$ and $B$ is given by the usual product $A B$ of subsets of a group, $A^{\smile}$ 
is given by $A^{-1}$, and $1^{\prime}$ is $\{e\}$. It follows that $\left(G^{+}\right)^{(1)}$ consists of all subgroups of $G$ and $\left(G^{+}\right)^{(2)}$ consists of all pairs of subgroups $(A, B)$ with $A \cap B$ equal to $\{e\}$ and $A B=G$. Notice that if $G$ is an abelian group, then $\left(G^{+}\right)^{(2)}$ is just Fact $G$.

Remark 4.11. We also have a way of constructing a relational orthomodular poset from any bounded modular lattice. For a bounded modular lattice $M$, let $M^{(2)}$ be the set of all ordered pairs of complementary elements of $M$, i.e. pairs $\left(x, x^{\prime}\right)$ with $x \cdot x^{\prime}=0$ and $x+x^{\prime}=1$. Define a relation $\subseteq$ on $M^{(2)}$ by setting $\left(x, x^{\prime}\right) \subseteq$ $\left(y, y^{\prime}\right)$ if $x \leq y$ and $y^{\prime} \leq x^{\prime}$, and define a unary operation $\perp$ on $M^{(2)}$ by setting $\left(x, x^{\prime}\right)^{\perp}$ to be $\left(x^{\prime}, x\right)$. The following theorem demonstrates that $M^{(2)}$ is a relational orthomodular poset. It is a pleasant exercise to give an elementary proof that $M^{(2)}$ is an orthomodular poset.

Mushtari [18] was the first to discover this construction, and was able to show somewhat more. Let $L$ be a lattice which is both $M$-symmetric and $M^{*}$-symmetric [16], and let $L^{(2)}$ be all pairs of complementary elements of $L$ which are both modular pairs and dual modular pairs [16]. Then $L^{(2)}$ forms an orthomodular poset with $\subseteq$ and $\perp$ as described above. ${ }^{1}$

Theorem 4.12. For a bounded modular lattice $M,\left(M^{(2)}, \subseteq, \perp,(0,1),(1,0)\right)$ is an orthomodular poset. Orthogonal joins in $M^{(2)}$ are given as follows:

$$
\text { if }\left(x, x^{\prime}\right) \subseteq\left(y, y^{\prime}\right)^{\perp} \text {, then }\left(x, x^{\prime}\right) \oplus\left(y, y^{\prime}\right)=\left(x+y, x^{\prime} \cdot y^{\prime}\right) .
$$

Further, for each bounded modular lattice $M$, there is a relation algebra $R_{M}$ with $M^{(2)}$ equal to $\left(R_{M}\right)^{(2)}$.

Proof. If $R$ is a commutative relation algebra (i.e. $a ; b=b ; a$ for all $a, b$ in $R$ ), then $\left(R^{(1)}, \leq, 1^{\prime}, 1\right)$ is a bounded modular lattice with meets agreeing with those in $R$, and joins being given by relational product. It is routine to see that $R^{(1)}$ is a lattice, and modularity follows from Lemma 2.2. It is apparent that for $M=\left(R^{(1)}, \leq, 1^{\prime}, 1\right)$, we have that $M^{(2)}$ is equal to $R^{(2)}$. For any bounded modular lattice $M$, define a ternary relation $S$ on $M$ by setting

$$
S=\{(x, y, z): x+y=x+z=y+z\} .
$$

Maddux [15] (see also [11]) has shown that the complex algebra of $(M, S,\{0\})$ is a symmetric $\left(x=x^{\smile}\right)$, hence commutative, relation algebra which we denote by $R_{M}$. Further, the modular lattice $\left(R_{M}\right)^{(1)}$ is the ideal lattice $\mathcal{I} M$ of $M$. As $M$ is a bounded modular lattice, the complemented elements of $\mathcal{I} M$ are exactly the principal ideals generated by complemented elements of $M$. Therefore, up to an obvious isomorphism, $\left(R_{M}\right)^{(2)}=(\mathcal{I} M)^{(2)}=M^{(2)}$.

Remark 4.13. For a modular ortholattice $M$, we can embed $M$ into $M^{(2)}$ via the map $x \leadsto\left(x, x^{\perp}\right)$. As this map preserves orthocomplementation and orthogonal joins, every modular ortholattice is a relational orthomodular poset.

Remark 4.14. One might hope that for a complete modular lattice $M$, the relational orthomodular poset $M^{(2)}$ would be orthocomplete. This is generally not the case. However, if $M$ is both join and meet continuous, then $M^{(2)}$ is orthocomplete. To verify this, suppose that $\left(x_{i}, x_{i}^{\prime}\right), i \in I$, is a pairwise orthogonal family of elements of $M^{(2)}$. For any finite subset $F$ of $I$, let $X_{F}=\sum_{F} x_{i}$ and $X_{F}^{\prime}=\prod_{F} x_{i}^{\prime}$. Clearly,

\footnotetext{
${ }^{1}$ Mushtari claims a stronger result [18, Theorem 2, p. 67], but this is not correct. See the third lattice of [16, Exercise 1.1, p. 5] for a counterexample.
} 
the ordered pair $\left(X_{F}, X_{F}^{\prime}\right)$ is equal to $\sum_{F}\left(x_{i}, x_{i}^{\prime}\right)$. Let $X=\sum_{I} x_{i}$ and $X^{\prime}=\prod_{I} x_{i}^{\prime}$. We have only to show that $X$ and $X^{\prime}$ are complements. But

$$
X^{\prime} \cdot X=X^{\prime} \cdot \sum_{I} x_{i}=X^{\prime} \cdot \sum_{F \subseteq I} X_{F}=\sum_{F \subseteq I}\left(X^{\prime} \cdot X_{F}\right) \leq \sum_{F \subseteq I}\left(X_{F}^{\prime} \cdot X_{F}\right)=0 .
$$

We have used continuity, and the fact that $\sum_{F \subseteq I} X_{F}$ is the join of an upwardly directed family. By symmetry, $X+X^{\prime}=1$.

We can apply these remarks to metric lattices [3], a class of lattices which includes finite modular lattices and continuous geometries. As the metric space completion of a metric lattice is both join and meet continuous [3], it follows that for a metric lattice $M$, the orthomodular poset $M^{(2)}$ can be embedded into an orthocomplete orthomodular poset. One can also check that for any metric lattice $M$, the orthomodular poset $M^{(2)}$ has a finitely additive state, and if $M$ is metrically complete, this state is countably additive.

\section{DeCOMPOSITIONS OF GROUPS AND MODULES}

In this section we apply our results on decompositions of algebras to groups with operators, a class of algebras that includes groups and modules.

Definition 5.1. Following van der Waerden [21, p. 138], a group with operators consists of a group $G$ together with a family $\mathcal{F}$ of endomorphisms of $G$, i.e. each $\alpha \in$ $\mathcal{F}$ is a unary map $\alpha: G \longrightarrow G$ which is compatible with the group multiplication, inverse, and identity. Any module is a group with operators, as is any group. Homomorphisms and subalgebras of a group $G$ with operators are defined in the usual manner, considering the operators in $\mathcal{F}$ to be fundamental operations of the algebra. We prefer to write the group operations as,+- and 0 , though a group with operators need not be abelian.

We begin by describing the various methods at hand to represent direct decompositions of a group $G$ with operators.

Definition 5.2. Let $G$ be a group with operators. We say that $G$ is the inner direct sum of $G_{1}, \ldots, G_{n}$, written $G=G_{1} \oplus \cdots \oplus G_{n}$, if (i) $G_{1}, \ldots G_{n}$ are subalgebras of $G$, (ii) every element $g \in G$ can be expressed in a unique fashion as a sum $g=g_{1}+\cdots+g_{n}$ where $g_{i} \in G_{i}$, and (iii) in each such unique representation $g=g_{1}+\cdots+g_{n}$, the elements $g_{1}, \ldots, g_{n}$ are pairwise commuting. For an inner direct sum $G=G_{1} \oplus G_{2}$ we define maps $\mu_{i}: G \longrightarrow G_{i}$ for $i=1,2$ by setting

(5.2.1) $\mu_{1} g=g_{1}$ and $\mu_{2} g=g_{2}$ if $g=g_{1}+g_{2}$ with $g_{1} \in G_{1}$ and $g_{2} \in G_{2}$.

We define the collection of all inner direct sums of $G$ by setting

(5.2.2) Sums $G=\left\{\left(G_{1}, G_{2}\right): G=G_{1} \oplus G_{2}\right\}$.

Definition 5.3. For a group $G$ with operators, we denote the collection of endomorphisms of $G$ by End $G$. We use 1 to denote the identity map on $G$ and 0 for the endomorphism which sends each element of $G$ to 0 . For endomorphisms $f$ and $g$, we define maps $f g$ and $f+g$ by setting $f g$ to be the composition of $f$ and $g$, and $f+g$ to be the pointwise sum of $f$ and $g$. Each such map $f g$ is an endomorphism of $G$, but $f+g$ need not be an endomorphism if $G$ is not abelian. We then set

(5.3.1) $E($ End $G)=\{f \in$ End $G: f=f f$ and $1-f=-f+1 \in$ End $G\}$. 
Note that if $G=G_{1} \oplus G_{2}$ then the map $\mu_{1}$ is in $E($ End $G)$, and $1-\mu_{1}=\mu_{2}$. If $G$ is an abelian group with operators, then $E$ (End $G$ ) has a particularly simple description - it is the collection of idempotents of the endomorphism ring of $G$.

The connection between decompositions of $G$ and End $G$ is partially furnished by the following theorem. The proof of this theorem is not difficult, but it is quite lengthy. We leave the details to the reader, and note that hints are given in [17, exercises 4-6, p. 293].

Theorem 5.4. Let $G$ be a group with operators. The maps in the following diagram are in pairs of mutually inverse isomorphisms, and all triangles in this diagram commute:

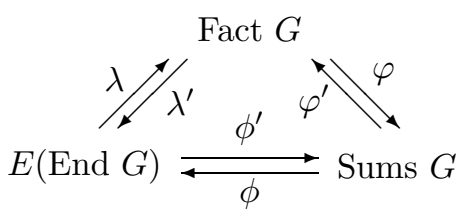

These maps are defined as follows;

(5.4.1) $\left(\alpha_{1}, \alpha_{2}\right) \stackrel{\varphi}{\sim}\left(0 / \alpha_{1}, 0 / \alpha_{2}\right)$,

(5.4.2) $\left(G_{1}, G_{2}\right) \stackrel{\varphi^{\prime}}{\rightarrow}\left(\alpha_{1}, \alpha_{2}\right)$, where $\alpha_{i}=\left\{(x, y): x-y \in G_{i}\right\}$,

(5.4.3) $\left(G_{1}, G_{2}\right) \stackrel{\phi}{\sim} \mu_{1}($ see $(5.2 .1))$,

(5.4.4) $f \stackrel{\phi^{\prime}}{\rightarrow}(f[G],(1-f)[G])$,

(5.4.5) $f \stackrel{\lambda}{\sim}(\operatorname{ker}(1-f), \operatorname{ker} f)$

(5.4.6) $\left(\alpha_{1}, \alpha_{2}\right) \stackrel{\lambda^{\prime}}{\rightarrow} f$, where $f x=y$ if $0 \alpha_{1} y \alpha_{2} x$.

As the sets Fact $G$, Sums $G$ and $E($ End $G$ ) are isomorphic and Fact $G$ is an orthomodular poset, the other sets also carry the structure of orthomodular posets. The next three theorems outline the details of the orthomodular structure on each of these sets. We use $\perp$ to denote orthocomplementation, $\leq$ for the partial ordering and $\oplus$ for orthogonal joins.

Theorem 5.5. Let $G$ be a group with operators. Then Fact $G$ is an orthomodular poset, where

(5.5.1) $\left(\alpha_{1}, \alpha_{2}\right)^{\perp}=\left(\alpha_{2}, \alpha_{1}\right)$

(5.5.2) $\left(\alpha_{1}, \alpha_{2}\right) \leq\left(\beta_{1}, \beta_{2}\right)$ if $\alpha_{1} \subseteq \beta_{1}$ and $\beta_{2} \subseteq \alpha_{2}$,

$(5.5 .3)\left(\alpha_{1}, \alpha_{2}\right) \oplus\left(\beta_{1}, \beta_{2}\right)=\left(\alpha_{1} ; \beta_{1}, \alpha_{2} \cdot \beta_{2}\right)$.

Proof. This follows immediately from the results of Section 3 taking into account that all congruences on a group permute.

Theorem 5.6. Let $G$ be a group with operators. Then Sums $G$ is an orthomodular poset, where

(5.6.1) $\left(G_{1}, G_{2}\right)^{\perp}=\left(G_{2}, G_{1}\right)$,

(5.6.2) $\left(G_{1}, G_{2}\right) \leq\left(H_{1}, H_{2}\right)$ if $G_{1} \subseteq H_{1}$ and $H_{2} \subseteq G_{2}$,

(5.6.3) $\left(G_{1}, G_{2}\right) \oplus\left(H_{1}, H_{2}\right)=\left(G_{1} \oplus H_{1}, G_{2} \cap H_{2}\right)$.

We use $G_{1} \oplus H_{1}$ to denote $\left\{g_{1}+h_{1}: g_{1} \in G_{1}\right.$ and $\left.h_{1} \in H_{1}\right\}$.

Proof. Recall that by Theorem 5.4, $\varphi$ and $\varphi^{\prime}$ are mutually inverse isomorphisms between Fact $G$ and Sums $G$. As $\varphi^{\prime}\left(G_{2}, G_{1}\right)=\varphi^{\prime}\left(G_{1}, G_{2}\right)^{\perp}$, it follows that $\left(G_{2}, G_{1}\right)$ is equal to $\varphi\left(\varphi^{\prime}\left(G_{1}, G_{2}\right)^{\perp}\right)$. The normal subgroup $G_{1}$ is contained in the normal 
subgroup $H_{1}$ if and only if the congruence associated with $G_{1}$ is contained in the congruence associated with $H_{1}$. Therefore $\varphi^{\prime}\left(G_{1}, G_{2}\right) \leq \varphi^{\prime}\left(H_{1}, H_{2}\right)$ if and only if $G_{1} \subseteq H_{1}$ and $H_{2} \subseteq G_{2}$. Finally, to verify that

$$
\varphi\left(\varphi^{\prime}\left(G_{1}, G_{2}\right) \oplus \varphi^{\prime}\left(H_{1}, H_{2}\right)\right)=\left(G_{1} \oplus H_{1}, G_{2} \cap H_{2}\right),
$$

it is sufficient to note that $G_{1} \oplus H_{1}$ is the normal subgroup generated by $G_{1} \cup H_{1}$.

Theorem 5.7. Let $G$ be a group with operators. Then $E(E n d G)$ is an orthomodular poset, where

(5.7.1) $f^{\perp}=1-f$,

(5.7.2) $f \leq g$ if $f g=f=g f$,

(5.7.3) $f \oplus g=f+g$.

In particular, $f$ is orthogonal to $g$ if and only if $f g=0=g f$.

Proof. Recall that by Theorem 5.4, $\phi$ and $\phi^{\prime}$ are mutually inverse isomorphisms between Sums $G$ and $E$ (End $G)$. As $\phi^{\prime}(1-f)=\left(\phi^{\prime} f\right)^{\perp}$, it follows that $1-f=$ $\phi\left(\phi^{\prime} f^{\perp}\right)$. Next, we note that as $g$ is idempotent, $g f=f$ if and only if $f[G] \subseteq g[G]$. Then, as $(1-f)(1-g)=1-g$ if and only if $f g=f$, it follows that $f g=f$ if and only if $(1-g)[G] \subseteq(1-f)[G]$. We have shown that $\phi^{\prime} f \leq \phi^{\prime} g$ if and only if $g f=f=f g$. Finally, we claim that if $f$ is orthogonal to $g$, then

$$
\phi\left(\phi^{\prime} f \oplus \phi^{\prime} g\right)=f+g .
$$

This follows from the observation that if $x=\left(x_{1}+x_{2}\right)+x_{3}$, with $x_{1}$ in $f[G], x_{2}$ in $g[G]$ and $x_{3}$ in $(1-f)[G] \cap(1-g)[G]$, then $(f+g) x=x_{1}+x_{2}$.

Remark 5.8. As the endomorphisms of a module $M$ form a ring, Theorem 5.7 shows that Fact $M$ is isomorphic to the orthomodular poset $\mathcal{L}($ End $M)$ of idempotents of the endomorphism ring of $M$. However, we have noted in Remark 4.9 that for any ring $A$, the orthomodular poset $\mathcal{L} A$ is isomorphic to Fact $A_{A}$. Therefore, the orthomodular posets which arise as $\mathcal{L} A$ for some ring $A$ are exactly the orthomodular posets which arise as decompositions of some module.

Remark 5.9. Jónsson has shown that one can describe the decompositions of any algebra with zero both in terms of inner direct sums and in terms of endomorphisms of the algebra [17, pp. 282-283]. For any algebra $G$ with zero, one could describe the orthomodular poset Fact $G$ both in terms of Sums $G$ and in terms of $E$ (End $G$ ), although a slightly more complicated definition of $E$ (End $G$ ) would be required. Of course, the results of Section 4 have provided a description of the orthomodular poset Fact $A$ for any type of algebraic, topological or relational structure $A$.

\section{Projections of NORMED GROUPS}

We continue our investigation of decompositions of groups and modules, but we add a topological element to the mix. Specifically, we define a norm on a group with operators, and show that a sensible definition can be made of a projection operator on such a normed group. Ultimately, we will show that the projection operators on a complete normed abelian group form a $\sigma$-complete orthomodular poset with a full set of countably additive states. These are the basic requirements of the quantum logic approach to quantum mechanics.

Definition 6.1. A norm on a group $G$ with operators is a map $\|\cdot\|$ from $G$ to the reals which satisfies 
(6.1.1) $\|x\| \geq 0$ for all $x \in G$,

(6.1.2) $\|x\|=0$ if and only if $x=0$,

(6.1.3) $\|x\|=\|-x\|$ for all $x \in G$,

(6.1.4) $\|x+y\| \leq\|x\|+\|y\|$ for all $x, y \in G$.

A strong norm is a norm $\|\cdot\|$ which also satisfies

(6.1.5) $\|x+y\|=\|y+x\|$ for all $x, y \in G$.

Definition 6.2. An inner direct sum $\left(G_{1}, G_{2}\right) \in \operatorname{Sums} G$ is a projection of $G$ if

(6.2.1) $\left\|g_{1}+g_{2}\right\|^{2}=\left\|g_{1}\right\|^{2}+\left\|g_{2}\right\|^{2}$ for all $g_{1} \in G_{1}$ and $g_{2} \in G_{2}$.

The set of all projections on $G$ is denoted by Proj $G$.

Lemma 6.3. Let $\left(G_{1}, G_{2}\right)$ and $\left(H_{1}, H_{2}\right)$ be projections on $G$.

(6.3.1) If $G_{1} \subseteq H_{1}$, then $H_{2} \subseteq G_{2}$.

(6.3.2) Projections are uniquely determined by their first components.

Proof. The second statement will follow trivially once we have established the first. Assume $\left(G_{1}, G_{2}\right)$ is a projection and $G_{1} \subseteq H_{1}$. For any $h_{2}$ in $H_{2}$, we can find $g_{1}$ in $G_{1}$ and $g_{2}$ in $G_{2}$ with $h_{2}=g_{1}+g_{2}$ and

$$
\left\|h_{2}\right\|^{2}=\left\|g_{1}\right\|^{2}+\left\|g_{2}\right\|^{2} .
$$

Then $g_{2}=-g_{1}+h_{2}$. But $G_{1} \subseteq H_{1}$ and $\left(H_{1}, H_{2}\right)$ is a projection, so

$$
\left\|g_{2}\right\|^{2}=\left\|-g_{1}\right\|^{2}+\left\|h_{2}\right\|^{2} .
$$

This gives us that

$$
\left\|g_{2}\right\|^{2}=\left\|-g_{1}\right\|^{2}+\left\|g_{1}\right\|^{2}+\left\|g_{2}\right\|^{2} .
$$

It follows that $g_{1}=0$, and therefore $h_{2}=g_{2}$ is an element of $G_{2}$.

For an inner direct sum $\left(G_{1}, G_{2}\right)$, each element $g \in G$ has a unique representation $g=g_{1}+g_{2}$ where $g_{1} \in G_{1}$ and $g_{2} \in G_{2}$. Given an inner direct sum $\left(G_{1}, G_{2}\right)$, we defined $\mu_{1} g=g_{1}$ if $g=g_{1}+g_{2}$ with $g_{1} \in G_{1}$ and $g_{2} \in G_{2}$. A subtle point is that the map $\mu_{1}$ depends not just on the summand $G_{1}$, but on both summands $G_{1}$ and $G_{2}$. This follows from the fact that the map $\mu_{1}$ completely determines the inner direct sum $\left(G_{1}, G_{2}\right)$ (see Theorem 5.4) and $G_{1}$ may be a direct summand of two different inner direct sums. If $\left(G_{1}, G_{2}\right)$ is a projection of $G$ we fare better, as the inner direct sum $\left(G_{1}, G_{2}\right)$ is completely determined by the summand $G_{1}$. It is therefore possible to recover the map $\mu_{1}$ from the summand $G_{1}$. This is the basis of the following definition.

Definition 6.4. Let $A$ be a direct summand of some projection of $G$. Then by (6.3.2), there is only one subalgebra $B$ with $(A, B)$ a projection of $G$. Each $g \in G$ can be represented in a unique fashion as the sum of an element $a \in A$ and an element $b \in B$. Therefore, we may define a map $\hat{A}: G \longrightarrow A$ by setting $\hat{A} g=a$. Note that $\hat{A}$ is just the map $\mu_{1}$ of (5.2.1), and therefore $\hat{A} \in E$ (End $G$ ). We define

$$
\text { ProjOpG }=\{\hat{A}:(A, B) \in \operatorname{Proj} G\}
$$

and call such maps $\hat{A}$ projection operators on $G$.

Lemma 6.5. Let $(A, B)$ be a projection on $G$. Then

(6.5.1) $\|x\|^{2}=\|\hat{A} x\|^{2}+\|\hat{B} x\|^{2}$ for all $x \in G$,

(6.5.2) $\|\hat{A} x\| \leq\|x\|$ for all $x \in G$,

(6.5.3) $\|\hat{A} x\|=\|x\|$ if and only if $x \in A$, 
(6.5.4) $\|\hat{A} x\|=0$ if and only if $x \in B$.

Proof. For each $x \in G$, the unique representation of $x$ as the sum of an element of $A$ and an element of $B$ is given by $x=\hat{A} x+\hat{B} x$. These statements then follow directly from the definition of a projection (6.2.1) and the fact that the norm of an element is 0 if and only if the element is 0 (6.1.2).

Lemma 6.6. Let $G$ be a normed group with operators. If $\left(A_{1}, B_{1}\right)$ and $\left(A_{2}, B_{2}\right)$ are orthogonal projections whose join in Sums $G$ is $(A, B)$, then

(6.6.1) $A=A_{1} \oplus A_{2}$ and $B=B_{1} \cap B_{2}$,

(6.6.2) $(A, B)$ is a projection,

(6.6.3) $\hat{A}=\hat{A}_{1}+\hat{A}_{2}=\hat{A}_{2}+\hat{A}_{1}$,

(6.6.4) $\hat{B}=\hat{B}_{1} \hat{B}_{2}=\hat{B}_{2} \hat{B}_{1}$.

Proof. The first assertion is a restatement of (5.6.3) For the second, if $a \in A$ and $b \in B$, there are elements $a_{1} \in A_{1}$ and $a_{2} \in A_{2}$ with $a=a_{1}+a_{2}$. As $\left(A_{1}, B_{1}\right)$ is orthogonal to $\left(A_{2}, B_{2}\right)$, we have that $A_{1} \subseteq B_{2}$ and $A_{2} \subseteq B_{1}$. Therefore $a_{2}+b \in B_{1}$ and $b \in B_{2}$, giving

$$
\|a+b\|^{2}=\left\|a_{1}+\left(a_{2}+b\right)\right\|^{2}=\left\|a_{1}\right\|^{2}+\left\|a_{2}+b\right\|^{2}=\left\|a_{1}\right\|^{2}+\left\|a_{2}\right\|^{2}+\|b\|^{2} .
$$

Then as $a_{2} \in B_{1}$ we have

$$
\|a+b\|^{2}=\left\|a_{1}+a_{2}\right\|^{2}+\|b\|^{2}=\|a\|^{2}+\|b\|^{2} .
$$

So $(A, B)$ is a projection. The third assertion is a restatement of (5.7.3). For the fourth statement, note first that by Theorem $5.7 \hat{A}_{1} \hat{A}_{2}=0=\hat{A}_{2} \hat{A}_{1}$ since $\left(A_{1}, B_{1}\right)$ and $\left(A_{2}, B_{2}\right)$ are orthogonal. Using the fact that $\hat{B}=1-\hat{A}, \hat{B}_{1}=1-\hat{A}_{1}$ and $\hat{B}_{2}=1-\hat{A}_{2}$, the fourth statement follows by a simple calculation.

Recall that a state on an orthomodular poset $P$ is a map $\varphi$ from $P$ to the real unit interval $[0,1]$ satisfying (i) $\varphi(0)=0$, (ii) $\varphi(1)=1$, and (iii) if $x$ is orthogonal to $y$, then $\varphi(x \oplus y)=\varphi(x)+\varphi(y)$. If every countable pairwise orthogonal set has a join in $P$, we call $P$ a $\sigma$-complete orthomodular poset. A state $\varphi$ on a $\sigma$-complete orthomodular poset $P$ is called countably additive if for every countable pairwise orthogonal family of elements $\left(x_{n}\right)_{n \in N}$, the sum $\sum_{1}^{\infty} \varphi\left(x_{n}\right)$ exists and is equal to $\varphi(x)$, where $x$ is the join of $\left(x_{n}\right)_{n \in N}$ in $P$. Finally, we say that a set $\mathcal{S}$ of states on an orthomodular poset $P$ is full if for all $x \not \leq y$ in $P$ there is a state $\varphi$ in $\mathcal{S}$ with $\varphi(x) \not \leq \varphi(y)$. See $[13,19]$ for further details.

Theorem 6.7. Let $G$ be a normed group with operators. Then Proj $G$ is a suborthomodular poset of Sums $G$. Further, $\left\{s_{g}: 0 \neq g \in G\right\}$ is a full set of finitely additive states on Proj $G$, where

$$
s_{g}(A, B)=\frac{\|\hat{A} g\|^{2}}{\|g\|^{2}} .
$$

Proof. Clearly Proj $G$ is closed under the orthocomplementation of Sums $G$, and the closure of Proj $G$ under orthogonal joins is given by (6.6.2). Therefore Proj $G$ is a sub-orthomodular poset of Sums $G$.

To verify that $s_{g}$ is a state, we first note that $s_{g}(\{0\}, G)=0$ and $s_{g}(G,\{0\})=1$. Suppose that $\left(A_{1}, B_{1}\right)$ is orthogonal to $\left(A_{2}, B_{2}\right)$, with their join being $(A, B)$. Then

$$
s_{g}\left(A_{1}, B_{1}\right)+s_{g}\left(A_{2}, B_{2}\right)=\frac{\left\|\hat{A}_{1} g\right\|^{2}+\left\|\hat{A}_{2} g\right\|^{2}}{\|g\|^{2}}=\frac{\left\|\hat{A}_{1} g+\hat{A}_{2} g\right\|^{2}}{\|g\|^{2}}=s_{g}(A, B) .
$$


Therefore each map $s_{g}$, with $g \neq 0$, is a finitely additive state on Proj $G$. To show that the collection of all such states is full, suppose $\left(A_{1}, B_{1}\right)$ and $\left(A_{2}, B_{2}\right)$ are projections and that $\left(A_{1}, B_{1}\right) \not \leq\left(A_{2}, B_{2}\right)$. Then by (6.3.1) we have that $A_{1} \nsubseteq A_{2}$. So there is a non-zero element $g$ in $A_{1}$ which is not in $A_{2}$. It follows by Lemma 6.5 that $s_{g}\left(A_{1}, B_{1}\right) \not z s_{g}\left(A_{2}, B_{2}\right)$.

To this point, we have made use only of algebraic properties of the norm. We will see that a norm induces a metric space topology on a group just as with vector spaces. Our strongest results will come by considering topological properties of projections and projection operators.

Lemma 6.8. Let $G$ be a normed group with operators.

(6.8.1) The map $d(x, y)=\|x-y\|$ is a metric on $G$.

(6.8.2) Under the topology induced by this metric, the norm is continuous.

(6.8.3) If the norm is a strong norm, the group operations are continuous.

(6.8.4) If $A$ is a direct summand of a projection, then $\hat{A}$ is continuous.

(6.8.5) If $A$ is a direct summand of a projection, then $A$ is closed.

Proof. The only non-trivial part of the first statement is the triangle inequality, which follows by (6.1.4) since

$$
d(x, z)=\|x-z\|=\|(x-y)+(y-z)\| \leq\|x-y\|+\|y-z\|=d(x, y)+d(y, z) .
$$

For the second statement, we must show that, for any Cauchy sequence $\left\{x_{n}\right\}$ in $G$ converging to $x,\left\{\left\|x_{n}\right\|\right\}$ converges to $\|x\|$. Note first that

$$
\|x\|-\left\|x_{n}\right\| \leq\left\|x-x_{n}\right\| .
$$

(This is derived from (6.1.4) using $x=\left(x-x_{n}\right)+x_{n}$.) Similarly

$$
\left\|x_{n}\right\|-\|x\| \leq\left\|x_{n}-x\right\|=\left\|x-x_{n}\right\|
$$

(This is derived from (6.1.3) and (6.1.4) using $x_{n}=\left(x_{n}-x\right)+x$.) Therefore

$$
d^{\prime}\left(\|x\|,\left\|x_{n}\right\|\right) \leq d\left(x, x_{n}\right)
$$

where $d^{\prime}$ is the usual metric on the reals. As $\left\{x_{n}\right\}$ converges to $x$, it follows that $\left\{\left\|x_{n}\right\|\right\}$ converges to $\|x\|$.

For the third statement we must show that, for Cauchy sequences $\left\{x_{n}\right\}$ and $\left\{y_{n}\right\}$ converging to $x$ and $y$ respectively, $\left\{-x_{n}\right\}$ converges to $-x$ and $\left\{x_{n}+y_{n}\right\}$ converges to $x+y$. That $\left\{-x_{n}\right\}$ converges to $-x$ follows as

$$
\left\|-x_{n}-(-x)\right\|=\left\|-x_{n}+x\right\|=\left\|x-x_{n}\right\|
$$

(note we have used the fact that $\|\cdot\|$ is a strong norm to obtain the last equality). Similarly, we have that $\left\{x_{n}+y_{n}\right\}$ converges to $x+y$ by considering

$$
\left\|\left(x_{n}+y_{n}\right)-(x+y)\right\|=\left\|\left(x_{n}+y_{n}-y\right)-x\right\|=\left\|\left(-x+x_{n}\right)+\left(y_{n}-y\right)\right\|
$$

(we have again used the fact that $\|\cdot\|$ is a strong norm to obtain the last equality). Now by applying (6.1.4) we have

$$
\left\|\left(x_{n}+y_{n}\right)-(x+y)\right\| \leq\left\|-x+x_{n}\right\|+\left\|y_{n}-y\right\|=\left\|x_{n}-x\right\|+\left\|y_{n}-y\right\|
$$

(we have again used the fact that $\|\cdot\|$ is a strong norm in obtaining the last equality), and it follows that $\left\{x_{n}+y_{n}\right\}$ converges to $x+y$. 
For the fourth statement we must show that for a Cauchy sequence $\left\{x_{n}\right\}$ converging to $x$, the sequence $\left\{\hat{A} x_{n}\right\}$ converges to $\hat{A} x$. This is given by the following identity obtained using (6.5.2) and the fact that $\hat{A}$ is an endomorphism:

$$
\left\|\hat{A} x_{n}-\hat{A} x\right\|=\left\|\hat{A}\left(x_{n}-x\right)\right\| \leq\left\|x_{n}-x\right\| .
$$

For the fifth statement, assume that $(A, B)$ is a projection and $\left\{a_{n}\right\}$ is a Cauchy sequence of points in $A$ which converges to $a$. As $\hat{B}$ is continuous, $\left\{\hat{B} a_{n}\right\}$ converges to $\hat{B} a$. So by (6.5.4) $\hat{B} a=0$, which implies that $a \in A$.

Lemma 6.9. Let $G$ be a normed group with operators and $\left(A_{n}, B_{n}\right)_{n \in N}$ be a countable family of pairwise orthogonal projections. For each element $g \in G$ define $g^{n}=\hat{A}_{1} g+\cdots+\hat{A}_{n} g$ and $g_{n}=\hat{B}_{1} \hat{B}_{2} \cdots \hat{B}_{n} g$. Then

(6.9.1) $g^{n}+g_{n}=g$ for each $n$,

(6.9.2) $\left\{g^{n}\right\}$ is a Cauchy sequence,

(6.9.3) $\left\{g_{n}\right\}$ is a Cauchy sequence,

(6.9.4) If $\left\{g_{n}\right\}$ converges, then $\lim g_{n} \in \bigcap_{N} B_{n}$.

Proof. The first statement follows by Lemma 6.6. For the second statement, note first that by (6.6.3) the elements $\hat{A}_{1} g, \hat{A}_{2} g, \ldots$ all commute. This implies that $g^{n}-g^{m}=\hat{A}_{m+1} g+\cdots+\hat{A}_{n} g$ for all $m<n$. But by Lemma 6.6

$$
\|g\|^{2}=\left\|g^{n}\right\|^{2}+\left\|g_{n}\right\|^{2},
$$

since $\left(A_{1}, B_{1}\right) \oplus \cdots \oplus\left(A_{n}, B_{n}\right)$ is a projection. But

$$
\|g\|^{2} \geq\left\|g^{n}\right\|^{2}=\sum_{i=1}^{n}\left\|\hat{A}_{i} g\right\|^{2} .
$$

This implies that $\sum_{1}^{\infty}\left\|\hat{A}_{i} g\right\|^{2}$ converges. Then, as

$$
\left\|g^{n}-g^{m}\right\|^{2}=\sum_{i=m+1}^{n}\left\|\hat{A}_{i} g\right\|^{2},
$$

it follows that the sequence $\left\{g^{n}\right\}$ is Cauchy.

To verify the third statement, note first that $g_{n}-g_{m}=-g^{n}+g^{m}$ for all $m, n$. This is easily derived from the fact that $g^{n}+g_{n}=g^{m}+g_{m}$. As all the elements $\hat{A}_{1} g, \hat{A}_{2} g, \ldots$ commute, it follows that $-g^{n}+g^{m}=-\left(g^{n}-g^{m}\right)$. Therefore

$$
\left\|g_{n}-g_{m}\right\|=\left\|g^{n}-g^{m}\right\|
$$

giving that $\left\{g_{n}\right\}$ is Cauchy. We now consider the final statement. By (6.5.3), it is enough to show that $\hat{B}_{k}\left(\lim g_{n}\right)=\lim g_{n}$ for each $k$. By (6.6.4) and the fact that $\hat{B}_{k}$ is idempotent, it follows that $\hat{B}_{k} g_{n}=g_{n}$ for all $n \geq k$. Therefore $\lim \hat{B}_{k} g_{n}=\lim g_{n}$, and our result follows from the continuity of $\hat{B}_{k}$.

For our strongest results we will consider normed groups with operators which are complete under the metric induced by the norm and have their group operations as well as their operators continuous. In view of (6.8.3), any complete strongly normed group with continuous operators, and therefore any complete normed abelian group with continuous operators, will satisfy these conditions. In particular, these results apply to any complete normed abelian group (without operators). 
Theorem 6.10. Let $G$ be a complete normed group with operators whose group operations and operators are continuous. Then Proj $G$ is an orthocomplete relational orthomodular poset with a full set of countably additive states.

Proof. By Theorem 6.7 we have that Proj $G$ is a relational orthomodular poset and that $\left\{s_{g}: 0 \neq g \in G\right\}$ is a full set of finitely additive states. We have only to show that Proj $G$ is orthogonally complete and that these states are countably additive.

We begin by showing that Proj $G$ is orthogonally complete. Let $\left(A_{i}, B_{i}\right)_{i \in I}$ be a pairwise orthogonal family of projections. Define $A$ to be the closure of the subalgebra generated by $\bigcup_{I} A_{i}$, and $B$ to be $\bigcap_{I} B_{i}$. We will show that $(A, B)$ is a projection. It will then follow from (6.8.5) that $(A, B)$ is the join of the family $\left(A_{i}, B_{i}\right)_{i \in I}$.

Note first that $A$ and $B$ are subalgebras of $G$. That $A$ is a subalgebra follows from the continuity of the group operations and operators, and it is trivial that $B$ is a subalgebra.

Next, we show that $A \cap B=\{0\}$. If $a \in A$, then there is a Cauchy sequence $\left\{a_{n}\right\}$ converging to $a$ with each $a_{n}$ in the subalgebra generated by $\bigcup_{I} A_{i}$. As the subalgebra generated by $\bigcup_{I} A_{i}$ consists of all finite sums of elements of $\bigcup_{I} A_{i}$, we may assume there is a countable subset $i_{1}, i_{2}, \ldots$ of $I$ with $a_{n} \in A_{i_{1}} \oplus \cdots \oplus A_{i_{n}}$ for each $n$. If $a$ is also in $B$, then as $\left(A_{i_{1}}, B_{i_{1}}\right) \oplus \cdots \oplus\left(A_{i_{n}}, B_{i_{n}}\right)$ is a projection, it follows that $\left\|a-a_{n}\right\|^{2}=\|a\|^{2}+\left\|a_{n}\right\|^{2}$. As the sequence $\left\{a_{n}\right\}$ converges to $a$, we must conclude that $\|a\|^{2}=0$ and therefore $a=0$.

We continue towards our goal of showing that $(A, B)$ is a projection by showing that $(A, B)$ is an inner direct sum. We must show that each $g \in G$ has a unique representation $g=a+b$ with $a \in A$ and $b \in B$, and in this representation $a$ and $b$ commute. Note that

$$
\|g\|^{2} \geq\left\|\hat{A}_{i_{1}} g\right\|^{2}+\cdots+\left\|\hat{A}_{i_{n}} g\right\|^{2}
$$

for any $i_{1}, \ldots, i_{n}$ in $I$. It follows that $\left\{i \in I: \hat{A}_{i} g \neq 0\right\}$ is at most countable. Let $i_{1}, i_{2}, \ldots$ be an enumeration of this set. Defining $g^{n}=\hat{A}_{i_{1}} g+\cdots+\hat{A}_{i_{n}} g$ and $g_{n}=$ $\hat{B}_{i_{1}} \hat{B}_{i_{2}} \cdots \hat{B}_{i_{n}} g$, we have by Lemma 6.9 that $\left\{g^{n}\right\}$ and $\left\{g_{n}\right\}$ are Cauchy sequences. As $G$ is complete these sequences converge, say $a=\lim g^{n}$ and $b=\lim g_{n}$. Clearly $a \in A$. By (6.9.4), $b \in B_{i}$ for each $i$ with $\hat{A}_{i} g \neq 0$. But if $\hat{A}_{j} g=0$, then $\hat{B}_{j} g=g$. Then using the fact that $\hat{B}_{j}$ commutes with each $\hat{B}_{i}(6.6 .4)$, we have that $\hat{B}_{j} g_{n}=g_{n}$ for each $n$. The continuity of $\hat{B}_{j}$ then implies that $\hat{B}_{j} b=b$, so $b \in B_{j}$. We have shown that $a \in A$ and $b \in B$. The continuity of the group operations and the fact that $g^{n}+g_{n}=g_{n}+g^{n}=g$ then imply

$$
a+b=\lim g^{n}+\lim g_{n}=\lim \left(g^{n}+g_{n}\right)=g
$$

and

$$
b+a=\lim g_{n}+\lim g^{n}=\lim \left(g_{n}+g^{n}\right)=g .
$$

Finally, the uniqueness of the representation follows from the fact that $A \cap B=\{0\}$. So $(A, B)$ is an inner direct sum.

To show that $(A, B)$ is a projection, it is sufficient to show that

$$
\|g\|^{2}=\|a\|^{2}+\|b\|^{2},
$$

where $g=a+b$ with $a \in A$ and $b \in B$. Using the continuity of the norm and the fact that

the result follows easily.

$$
\|g\|^{2}=\left\|g^{n}\right\|^{2}+\left\|g_{n}\right\|^{2},
$$


To conclude the proof of the theorem, we have only to show that the state $s_{g}$ is countably additive. Suppose that $\left(A_{n}, B_{n}\right)_{n \in N}$ is a countable family of pairwise orthogonal projections whose join is $(A, B)$. As we have seen,

$$
\hat{A} g=\lim _{n \rightarrow \infty}\left(\hat{A_{1}} g+\cdots+\hat{A_{n}} g\right) \text {. }
$$

It follows from the continuity of the norm that

$$
\|\hat{A} g\|^{2}=\lim _{n \rightarrow \infty}\left\|\hat{A}_{1} g+\cdots+\hat{A}_{n} g\right\|^{2}=\lim _{n \rightarrow \infty}\left(\left\|\hat{A}_{1} g\right\|^{2}+\cdots+\left\|\hat{A}_{n} g\right\|^{2}\right)=\sum_{n=1}^{\infty}\left\|\hat{A_{n}} g\right\|^{2} .
$$

Therefore $s_{g}(A, B)=\sum_{1}^{\infty} s_{g}\left(A_{n}, B_{n}\right)$.

Remark 6.11. It seems that we have a sensible definition of projection operators for any normed group $G$ with operators. Special cases of normed groups with operators are of course Hilbert spaces. If $H$ is a real or complex Hilbert space, we claim that Proj $H$ consists of exactly those pairs $\left(A, A^{\perp}\right)$ such that $A$ is a closed subspace of $H$. It is easy enough to check that any such pair is in Proj $H$. But if $(A, B)$ is in Proj $H$, then $A$ is a closed subspace of $H$, and as projections are determined by their first components (6.3.2), it follows that $B=A^{\perp}$.

Remark 6.12. We have defined projections to be decompositions $(A, B)$ such that

$$
f(\|a+b\|)=f(\|a\|)+f(\|b\|) \text { for every } a \text { in } A \text { and } b \text { in } B,
$$

where $f(x)=x^{2}$. Our proofs have only used the properties that (i) $f(0)=0$; (ii) $f$ is strictly increasing; and (iii) $f$ is continuous. For any such function $f$, we could define $\operatorname{Proj}_{f} G$. All of our results are valid in this more general setting.

\section{Properties of Relational orthomodular posets}

In this section, we will show that there are orthomodular posets which are not relational orthomodular posets. We prove the stronger result that there is a finite orthomodular poset $P$ which has no mapping into a non-trivial relational orthomodular poset which preserves orthocomplementation and orthogonal joins. We further show that this orthomodular poset $P$ has no mapping into a non-trivial orthomodular lattice which preserves orthocomplementation and orthogonal joins. Surprisingly, it seems to have been an open question whether every orthomodular poset could be embedded into an orthomodular lattice.

Before we reach the main results of this section, we must prove a few technical details about relation algebras. It will be convenient to introduce some new notation to avoid repeating the same phrases endlessly.

Definition 7.1. We define a partial binary operation $\hat{j}$ on a relation algebra $R$. The operation $\hat{j}$ is to be defined for all pairs of proper equivalence elements $p, q$ for which $p ; q=q ; p$, and for such a pair, $p \hat{;} q$ is defined to be $p ; q$. Thus, for elements $p$ and $q$ of a relation algebra $R$, the notation $p \hat{;} q$ is understood to mean that $p$ and $q$ are proper equivalence elements with $p ; q$ equal to $q ; p$, and that the element $p \hat{;} q$ is equal to $p ; q$. Note that if $p \hat{;} q$ is defined, then $p \hat{;} q$ is also a proper equivalence element of $R$.

Lemma 7.2. Let $R$ be a relation algebra with $r \hat{;} t_{1}, r \hat{;} t_{2}, r \hat{;} q$ and $s \hat{;} p$.

(7.2.1) If $t_{1}, t_{2} \leq r^{\prime}$, where $r^{\prime} \in R^{(1)}$ with $r \cdot r^{\prime}=1^{\prime}$, then $\left(r ; t_{1}\right) \cdot\left(r \hat{;} t_{2}\right)=r \hat{;}\left(t_{1} \cdot t_{2}\right)$.

(7.2.2) If $p \leq q, r \leq s$ and $s \cdot q=1^{\prime}$, then $(r \hat{;} q) \cdot(s \hat{;} p)=r \hat{;} p$. 
Proof. To verify the first statement, let $a=\left(r \hat{;} t_{1}\right) \cdot\left(r ; t_{2}\right)$ and $b=r^{\prime}$ and $c=\left(t_{1} \cdot t_{2}\right) ; r$. Note that $c \leq a$ and that $a$ is a proper equivalence element, so we may apply (2.2.1) to the elements $a, b$ and $c$. This gives us that $a \cdot(b ; c)=(a \cdot b) ; c$. Noting that $a \leq b ; c$, we have that $a \cdot(b ; c)=a$. By applying $(2.2 .4)$ we have that $b \cdot\left(t_{i} \hat{;} r\right)$ equals $t_{i}$ for $i=1,2$. It follows that $a \cdot b$ equals $t_{1} \cdot t_{2}$ and therefore $(a \cdot b) ; c=c$. Having shown that $a=c$, it remains only to show that $\left(t_{1} \cdot t_{2}\right) ; r$ is equal to $r ;\left(t_{1} \cdot t_{2}\right)$. This follows as $\left(t_{1} \cdot t_{2}\right) ; r$ is equal to the proper equivalence element $a$.

To verify the second statement, let $a=(r \hat{;} q) \cdot(s \hat{;} p)$ and $b=s$ and $c=r ; p$. Note that $c \leq a$ and that $a$ is a proper equivalence element, so we may apply (2.2.1) to the elements $a, b$ and $c$. This gives us that $a \cdot(b ; c)=(a \cdot b) ; c$. Noting that $a \leq b ; c$, we have that $a \cdot(b ; c)=a$. By applying $(2.2 .4)$ we have that $s \cdot(r \hat{;} q)=r$. It follows that $(a \cdot b) ; c=c$. That $r ; p=p ; r$ follows as $r ; p$ is equal to the proper equivalence element $a$.

The following lemma states a few well-known facts about the arithmetic of orthomodular lattices [10]. For elements $a, b$ in an orthomodular lattice $L$, we use the notation $a \oplus b$ to mean both that $a \leq b^{\prime}$ and that $a \oplus b$ is the join of $a$ and $b$.

Lemma 7.3. Let $L$ be an orthomodular lattice, with $r \oplus t_{1}, r \oplus t_{2}, r \oplus q$ and $s \oplus p$.

(7.3.1) $\left(r \oplus t_{1}\right) \cdot\left(r \oplus t_{2}\right)=r \oplus\left(t_{1} \cdot t_{2}\right)$.

(7.3.2) If $p \leq q, r \leq s$ and $s \cdot q=0$, then $(r \oplus q) \cdot(s \oplus p)=r \oplus p$.

For the remainder of this section, we shall be concerned with the orthomodular posets of Figure 1 and Figure 2 below. We shall call the orthomodular poset of Figure 1 the orthomodular kite and the orthomodular poset of Figure 2 the double kite. These diagrams are called Greechie diagrams, and a complete account of Greechie diagrams is given in [13]. We review a few basic facts.

The dots in the diagrams represent atoms, and orthogonal atoms are joined by a straight line. Let $x$ and $y$ be orthogonal atoms, with $z$ the third element in the line containing $x$ and $y$. Then for an atom $w$, we have $w \leq x \oplus y$ if and only if $w \neq z$ and $w$ and $z$ are joined by a straight line. As each of these diagrams contains a four-loop, and neither contains a triangle, Greechie's loop lemma [9] implies that both these diagrams represent orthomodular posets which are not orthomodular lattices.

Definition 7.4. Let $R$ be a relation algebra and $\psi$ a map from an orthomodular poset $P$ to $R^{(2)}$ which preserves orthocomplementation and finite orthogonal joins. As elements of $R^{(2)}$ are ordered pairs of proper equivalence elements of $R$, we can define maps $\psi_{1}$ and $\psi_{2}$ from $P$ into $R^{(1)}$ by setting

(7.4.1) $\psi_{1}(x)=a$ and $\psi_{2}(x)=b$ if $\psi(x)=(a, b)$.

Note that if $x$ and $y$ are orthogonal elements of $P$, then

(7.4.2) $\psi_{1}(x \oplus y)=\psi_{1}(x) \hat{;} \psi_{1}(y)$.

Lemma 7.5. Let $R$ be a relation algebra. If $\psi$ is a map from the orthomodular kite of Figure 1 to $R^{(2)}$ which preserves orthocomplementation and finite orthogonal joins, and $\phi$ is a map from the double kite of Figure 2 to $R^{(2)}$ which preserves orthocomplementation and finite orthogonal joins, then

(7.5.1) $\psi_{1}(a) \leq \psi_{1}(c)$,

(7.5.2) $\phi(a)=0$. 


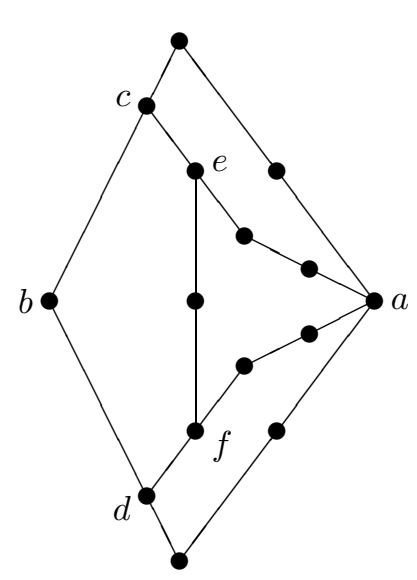

Figure 1

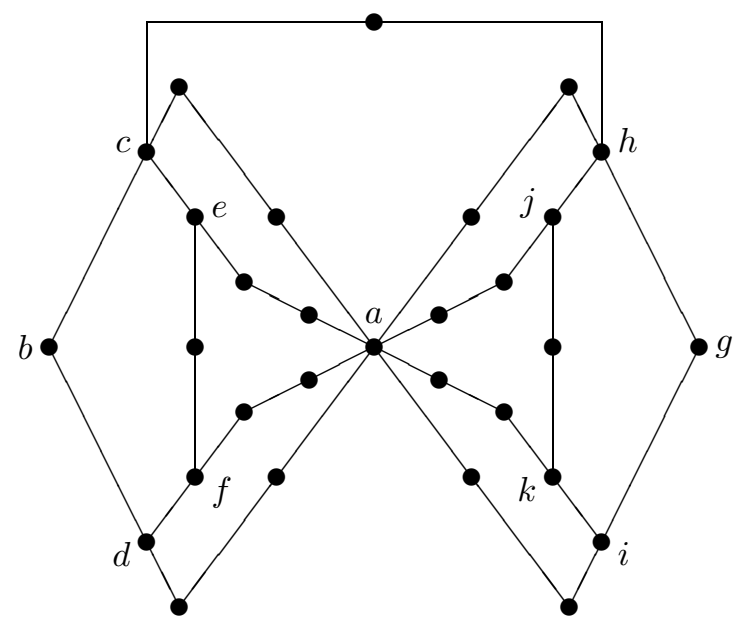

FIGURE 2

Proof. Note first that $\psi$ is necessarily order preserving, as $x \leq z$ in $P$ implies the existence of $y$ with $x \oplus y=z$. From the definition of the partial ordering in $R^{(2)}$, we have that $\psi_{1}$ is also order preserving. To improve the legibility of the following expressions, we shall temporarily adopt the convention of writing $\psi_{1}(x)$ as $x_{1}$ and $\psi_{2}(x)$ as $x_{2}$.

As $a$ lies beneath each of $b \oplus c, b \oplus d, c \oplus e$ and $d \oplus f$, we have that $a_{1}$ lies beneath each of $(b \oplus c)_{1}$, etc. It follows from (7.4.2) that $(b \oplus c)_{1}=b_{1} \hat{;} c_{1}$. So

$$
a_{1} \leq\left(b_{1} \hat{;} c_{1}\right) \cdot\left(b_{1} \hat{;} d_{1}\right) \cdot\left(c_{1} \hat{;} e_{1}\right) \cdot\left(d_{1} \hat{;} f_{1}\right) .
$$

As $c$ is orthogonal to $b$, we have that $c_{1} \leq b_{2}$, and similarly $d_{1} \leq b_{2}$. Then as $b_{2}$ is a proper equivalence element with $b_{1} \cdot b_{2}=1^{\prime}$, we may apply (7.2.1) to obtain that $\left(b_{1} \hat{;} c_{1}\right) \cdot\left(b_{1} \hat{;} d_{1}\right)$ is equal to $b_{1} \hat{;}\left(c_{1} \cdot d_{1}\right)$. Applying this idea several times to the last inequality, we obtain

$$
a_{1} \leq\left[b_{1} \hat{;}\left(c_{1} \cdot d_{1}\right)\right] \cdot\left[c_{1} \hat{;}\left(b_{1} \cdot e_{1}\right)\right] \cdot\left[d_{1} \hat{j}\left(b_{1} \cdot f_{1}\right)\right] .
$$

Then as $b_{1} \cdot e_{1} \leq b_{1}$ and $c_{1} \cdot d_{1} \leq c_{1}$ and $b_{1} \cdot c_{1}=1^{\prime}$ (since $c_{1} \leq b_{2}$ ), we may apply (7.2.2) to obtain that

$$
\left[b_{1} \hat{;}\left(c_{1} \cdot d_{1}\right)\right] \cdot\left[c_{1} \hat{;}\left(b_{1} \cdot e_{1}\right)\right]=\left(c_{1} \cdot d_{1}\right) \hat{;}\left(b_{1} \cdot e_{1}\right) .
$$

Applying this idea twice to the last inequality, we obtain

$$
a_{1} \leq\left[\left(c_{1} \cdot d_{1}\right) \hat{;}\left(b_{1} \cdot e_{1}\right)\right] \cdot\left[\left(c_{1} \cdot d_{1}\right) \hat{;}\left(b_{1} \cdot f_{1}\right)\right] .
$$

However, $b_{1} \cdot e_{1} \leq b_{1}$, and $b_{1} \cdot f_{1} \leq b_{1}$ and $b_{1}$ is a proper equivalence element with $b_{1} \cdot\left(c_{1} \cdot d_{1}\right)=1^{\prime}$ (since $\left.c_{1} \leq b_{2}\right)$. Therefore we may apply (7.2.1) to obtain

$$
a_{1} \leq\left(c_{1} \cdot d_{1}\right) \hat{;}\left(b_{1} \cdot e_{1} \cdot f_{1}\right) .
$$

But $b_{1} \cdot e_{1} \cdot f_{1}=1^{\prime}$ (since $f_{1} \leq e_{2}$ ), and the first statement is established.

For the second statement, we may apply (7.5.1) twice to obtain that $\phi_{1}(a) \leq$ $\phi_{1}(c)$ and $\phi_{1}(a) \leq \phi_{1}(h)$. But $c$ and $h$ are orthogonal, and therefore $\phi_{1}(a)=1^{\prime}$. But the only element of $R^{(2)}$ having $1^{\prime}$ as its first component is 0 . Therefore $\phi(a)=0$. 
Lemma 7.6. Let $L$ be an orthomodular lattice. If $\psi$ is a map from the orthomodular kite of Figure 1 to L which preserves orthocomplementation and finite orthogonal joins, and $\phi$ is a map from the double kite of Figure 2 to $L$ which preserves orthocomplementation and finite orthogonal joins, then

(7.6.1) $\psi(a) \leq \psi(c)$,

(7.6.2) $\phi(a)=0$.

Proof. The proof is similar to that of Lemma 7.6, replacing $\hat{j}$ with $\oplus$ and using (7.3.1) and (7.3.2) in place of (7.2.1) and (7.2.2) respectively.

Theorem 7.7. There is a finite orthomodular poset $P$ with the following properties: there is no map from $P$ into a non-trivial relational orthomodular poset which preserves orthocomplementation and orthogonal joins, and there is no map from $P$ into a non-trivial orthomodular lattice which preserves orthocomplementation and orthogonal joins.

Proof. Let $P$ be the orthomodular poset whose Greechie diagram is formed by taking three disjoint copies of the double kite, with middle elements $a, a^{\prime}$ and $a^{\prime \prime}$, and adding a line connecting $a, a^{\prime}$ and $a^{\prime \prime}$. If $R$ is a relation algebra and $\phi$ is a map from $P$ to $R^{(2)}$ which preserves orthocomplementation and orthogonal joins, then by (7.5.2) we have that $\phi(a), \phi\left(a^{\prime}\right)$ and $\phi\left(a^{\prime \prime}\right)$ are all equal to 0 . But $a, a^{\prime}$ and $a^{\prime \prime}$ are pairwise orthogonal, and their join is 1 . As $\phi$ preserves orthogonal joins, we have that the join of $\phi(a), \phi\left(a^{\prime}\right)$ and $\phi\left(a^{\prime \prime}\right)$ in $R^{(2)}$ must also be 1 . Therefore in $R^{(2)}, 0$ is equal to 1 , so $R^{(2)}$ is the trivial orthomodular poset. The corresponding result for orthomodular lattices clearly follows along similar lines, using (7.6.2) in place of (7.5.2).

One can present a superficial argument that the orthomodular kite depicted in Figure 1 could not represent the events of any quantum mechanical system. Suppose that the event labeled $a$ were to occur. We claim that this implies that the event $c$ must occur. We argue by contradiction. Suppose that the event $c$ does not occur. Then as $b \oplus c \geq a$, we have that $b$ must occur, and therefore $d$ does not occur. Then as $d \oplus f \geq a$, we can conclude that the event $f$ must occur. But $c \oplus e \geq a$, so the event $e$ must also occur. But it is not possible to have two orthogonal events both occur.

There are many holes in this argument, but it may contain a kernel of truth. The classical description of quantum mechanics is given by the orthomodular lattice of projection operators of a Hilbert space. As we have seen in Theorem 7.7, the orthomodular poset of Figure 1 cannot be embedded into this (or any other) orthomodular lattice.

\section{Conclusions}

I believe that the solution of the following problems would be a significant step in developing the theory of relational orthomodular posets.

Problem 1. Describe those categories $\mathcal{C}$ for which one can construct an orthomodular poset from the decompositions of an object $A$ in $\mathcal{C}$.

It is easily seen that some restrictions must be placed on $\mathcal{C}$ by considering a poset $P$ to be the category $\mathcal{C}$. Define a category $\mathcal{C}$ to be honest if (i) $\mathcal{C}$ is closed under finite (including empty) products and (ii) for every product diagram $(f, g, h)$ in $\mathcal{C}$, 
the diagram $\left(f \times h, g \times h, \pi_{2}, \pi_{2}\right)$ is a pushout. All the structures we have considered in this paper form honest categories. As a partial solution to Problem 1, I have been able to show that the decompositions of any object in an honest category form an orthoalgebra [8].

Problem 2. Do the projections of a complete strongly normed group $G$ form an orthomodular lattice?

This seems to be related to the following question. If $\hat{A}$ and $\hat{B}$ are projection operators on $G$, is the sequence $\left\{(\hat{A} \hat{B})^{n} g\right\}$ a Cauchy sequence for each $g \in G$ ?

Problem 3. Is every orthomodular lattice a relational orthomodular poset ?

Note that by Remark 4.13, every modular ortholattice is a relational orthomodular poset. Also, the argument used to produce an orthomodular poset $P$ which was not relational transferred nearly verbatim to show that $P$ could not be embedded into an orthomodular lattice.

Problem 4. Do decompositions provide a link between the quantum logic approach to quantum mechanics and the many world approach to quantum mechanics $[6]$ ?

In conclusion, I would like to thank Bjarni Jónsson and Michael Roddy. B. Jónsson pointed out the similarity between orthoalgebras and the idempotents of the endomorphism ring of an algebra with zero. His suggestion was the starting point of this investigation. A series of discussions with Michael Roddy was the basis of the results of Section 7 .

\section{REFERENCES}

1. I. Amemiya and H. Araki, A remark on Piron's paper, Publ. Res. Inst. Math. Sci., Sect. A 12 (1966), 423-427. MR 35:4130

2. L. Beran, Orthomodular Lattices, Algebraic Approach, Academia, Prague, and Reidel, Dordrecht, 1984. MR 86m:06015a

3. G. Birkhoff, Lattice Theory, Third ed., Amer. Math. Soc. Colloq. Publ. XXV, Providence, RI, 1967. MR 37:2638

4. S. Burris and H.P. Sankappanavar, A Course in Universal Algebra, Springer, 1981. MR 83k:08001

5. L. H. Chin and A. Tarski, Distributive and modular laws in relation algebras, Univ. of Calif. Publ. in Math. N.S. 1 (1951), 341-383. MR 13:312f

6. B. S. DeWitt and R. D. Graham (ed.), The Many-Worlds Interpretation of Quantum Mechanics, Princeton University Press, 1973.

7. J. Flachsmeyer, A note on orthocomplemented posets, Proc. Conf. Topology and Measure, Part III, Wissl Beitr. Ernst-Moritz-Arndt Univ., Greifswald (1982), 65-75. MR 83m:28001

8. D. J. Foulis, R. J. Greechie and G. T. Rüttimann, Filters and supports in orthoalgebras, Internat. J. Theoret. Phys. 31 (1992), 789-807. MR 93c:06014

9. R. J. Greechie, Orthomodular lattices admitting no states, J. Combin. Theory 10 (1971), 119-132. MR 43:120

10. R. J. Greechie, On generating distributive sublattices of orthomodular lattices, Proc. Amer. Math. Soc. 67 (1977), 17-22; 76 (1979), 216-218. MR 56:8454; MR 80f:06008

11. B. Jónsson, Varieties of relation algebras, Algebra Universalis 15 (1982), 273-298. MR 84g:08023

12. B. Jónsson and A. Tarski, Direct Decompositions of Finite Algebraic Systems, Notre Dame Math. Lectures, 5, South Bend, IN, 1947. MR 8:560b

13. G. Kalmbach, Orthomodular Lattices, Academic Press, 1983. MR 85f:06012

14. F. Katrnoška, Logics of idempotents of rings, Proc. Second Winter School on Measure Theory Ján Liptovský, (1990) Jednota Slovensk. Mat. Fyz., Bratislava, 1990, pp. 100-104. MR 92b:00045 
15. R. Maddux, Embedding modular lattices in relation algebras, Algebra Universalis 12 (1981), 242-246. MR 82d:06007

16. F. Maeda and S. Maeda, Theory of Symmetric Lattices, Springer, 1970. MR 44:123

17. R. McKenzie, G. McNulty and W. Taylor, Algebras, Lattices, Varieties, vol. 1, Wadsworth and Brooks, 1987. MR 883:08001

18. D. Kh. Mushtari, Projection logics in Banach spaces, Soviet Math. (Iz. VUZ) 33 (1989), 59-70. MR 91e: 46087

19. P. Pták and S. Pulmannová, Orthomodular Structures as Quantum Logics, Kluwer, Dordrecht, 1991. MR 94d:81018b

20. A. Tarski, On the calculus of binary relations, J. Symbolic Logic 6 (1941), 73-89. MR 3:130e

21. B. L. van der Waerden, Modern Algebra, English translation, 2 vols., Frederick Ungar Publishing Co., New York, 1949. MR 10:587b

Department of Mathematics, Brandon University, Brandon, Manitoba, R7A 6A9, CANADA

E-mail address: Harding@Buster.BrandonU.Ca 